\title{
REDUCTION APPROACHES FOR VIBRATION CONTROL OF REPETITIVE STRUCTURES *
}

\author{
CHEN Wei-min (陈伟民 $)^{1,3}$, SUN Dong-chang (孙东昌 $)^{2}$, WANG Da-jun (王大钧 $)^{1}$ \\ WEI Jian-ping (魏建萍 $)^{1}, \quad$ TONG Li-yong (全力勇 $)^{2}, \quad$ WANG Quan $(\text { 王泉 })^{4}$ \\ (1. State Key Laboratory for Turbulence and Complex System/Department of Mechanics \\ and Engineering Science, Peking University, Beijing 100871, P. R. China; \\ 2. School of Aerospace, Mechanical and Mechatronic Engineering, \\ University of Sydney, Sydney, NSW2006, Australia; \\ 3. Division of Engineering Sciences, Institute of Mechanics, Chinese Academy of Sciences, \\ Beijing 100080, P. R. China; \\ 4. Mechanical, Materials and Aerospace Engineering Department, \\ University of Central Florida, Orlando, FL 32816, USA) \\ (Communicated by YE Qing-kai)
}

\begin{abstract}
The reduction approaches are presented for vibration control of symmetric, cyclic periodic and linking structures. The condensation of generalized coordinates, the locations of sensors and actuators, and the relation between system inputs and control forces are assumed to be set in a symmetric way so that the control system posses the same repetition as the structure considered. By employing proper transformations of condensed generalized coordinates and the system inputs, the vibration control of an entire system can be implemented by carrying out the control of a number of sub-structures, and thus the dimension of the control problem can be significantly reduced.
\end{abstract}

Key words: vibration control of structures; repetitive structure; symmetric structure; cyclic periodic structure; linking structure

Chinese Library Classification: O32

2000 Mathematics Subject Classification: 74M05

Digital Object Identifier(DOI): 10.1007/s 10483-006-0509-z

\section{Introduction}

There are many repetitive structures in aeronautic, astronautic, civil and mechanical engineering applications, such as mirror-symmetric structures (symmetric structures for short), cyclic periodic structures, linear periodic structures, and linking structures. Repetitive structures are assembled with identical sub-structures in accordance with specified regulations. All of the sub-structures possesses the same geometric shape, physical properties, boundary conditions, and mutual influence. Due to these characteristics of repetitive structures, the natural vibration, forced vibration and vibration control can be solved based on the information from one single sub-structure alone. Therefore, the cost of numerical analysis and (or) experiments can be dramatically reduced.

Numerous studies have been made on the natural frequencies and vibration modes of repetitive structures. For instance, Evensen ${ }^{[1]}$ studied the vibration of symmetric structures.

* Received Oct.10, 2004; Revised Jan.12, 2005

Project supported by the National Natural Science Foundation of China (No.60034010) and the Australia Research Council Discovery-Projects Grant (No.DP0210716)

Corresponding author CHEN Wei-min, Doctor, E-mail: wmchen@imech.ac.cn 
Thomas ${ }^{[2]}$ investigated the vibration of cyclic periodic structures. Cai, Chan and Cheung ${ }^{[3,4]}$ conducted a series of research on the vibration of cyclic periodic structures by means of the Utransformation. Wang and Wang ${ }^{[5,6]}$ developed a reduction approach for symmetric structures and linking structures.

To the authors' knowledge, the vibration control of repetitive structures has not been widely studied so far. Bryson and Wiesinger ${ }^{[7]}$ provided an approach of a low order controller for vibration control of a circular flat plate space structure, in which a four-input four-output system was decoupled into four single-input single-output subsystems by utilizing geometric symmetry of the structure. The objective of this paper is to propose reduction approaches for vibration control of mirror-symmetric structures, cyclic periodic structures and linking structures. It is proved that the vibration control problem of a repetitive structure can be simplified into the vibration control problem of a number of sub-structures provided their generalized coordinate condensation, sensor and actuator locations, and relation between inputs and control forces also have the same repetitive properties as the structure itself. Therefore, the dimension of the control system can be greatly reduced.

\section{Reduction Approaches for Vibration Control of Mirror-Symmetric Struc- tures}

When the geometric shape, physical properties (e.g., Young's modules, Poisson ratio and density) and boundary conditions of a structure are symmetric with respect to a plane, this structure is called a mirror-symmetric structure, or symmetric structure for short, while the plane is called the symmetric plane. The generalized coordinates set on the symmetric plane, in the left part and in the right part are denoted by vectors $\boldsymbol{x}_{2}, \boldsymbol{x}_{1}$ and $\boldsymbol{x}_{3}$, respectively; whilst their dimensions are denoted by $b, p$ and $p$, respectively. Then, the governing equation of motion under the action of control force is

$$
M \ddot{x}+K \boldsymbol{x}+\boldsymbol{B u}=\mathbf{0},
$$

where $\boldsymbol{x}=\left[\boldsymbol{x}_{1}, \boldsymbol{x}_{2}, \boldsymbol{x}_{3}\right]^{\mathrm{T}}$ is a $(2 p+b)$-dimensional generalized coordinate vector, $\boldsymbol{u}$ denotes the input to the system, $\boldsymbol{B} \boldsymbol{u}$ is the control force. If the locations and labeling of the generalized coordinate systems are symmetric with respect to the symmetric plane, the stiffness matrix $\boldsymbol{K}$ can be expressed in a special block form as

$$
\boldsymbol{K}=\left[\begin{array}{ccc}
\boldsymbol{K}_{11} & \boldsymbol{K}_{12} & \boldsymbol{K}_{13} \\
\boldsymbol{K}_{12}^{\mathrm{T}} & \boldsymbol{K}_{22} & \boldsymbol{K}_{12}^{\mathrm{T}} \boldsymbol{S}_{p} \\
\boldsymbol{K}_{13}^{\mathrm{T}} & \boldsymbol{S}_{p} \boldsymbol{K}_{12} & \boldsymbol{K}_{11}^{\mathrm{T}}
\end{array}\right], \quad \boldsymbol{S}_{p}=\left[\begin{array}{cc} 
& 1 \\
&
\end{array}\right]
$$

where the superscript $\mathrm{T}$ denotes the transpose of a matrix, and the superscript $\tilde{\mathrm{T}}$ denotes the transpose of a matrix with respect to the second diagonal entities in the matrix, i.e., for a $(n \times k)$-matrix $\boldsymbol{A}=\left[a_{i j}\right], \boldsymbol{A}^{\tilde{\mathrm{T}}}=\left[a_{(n-j+1)(k-i+1)}\right]=\boldsymbol{S}_{k} \boldsymbol{A}^{\mathrm{T}} \boldsymbol{S}_{n}$. In matrix $\boldsymbol{K}, \boldsymbol{K}_{11}^{\mathrm{T}}=\boldsymbol{K}_{11}$, $\boldsymbol{K}_{13}^{\tilde{\mathrm{T}}}=\boldsymbol{K}_{13}, \boldsymbol{K}_{22}^{\mathrm{T}}=\boldsymbol{K}_{22}$. Mass matrix $\boldsymbol{M}$ has the same form as matrix $\boldsymbol{K}$ in the above sense.

When the actuators are located on the symmetric plane and in the left and right parts symmetrically, the input $\boldsymbol{u}$ of the control force consists of three components, i.e., two $m$ dimensional vectors for $\boldsymbol{u}_{1}, \boldsymbol{u}_{3}$ and one $s$-dimensional vector $\boldsymbol{u}_{2}$, namely, $\boldsymbol{u}=\left[\boldsymbol{u}_{1}, \boldsymbol{u}_{2}, \boldsymbol{u}_{3}\right]^{\mathrm{T}}$, with $m \leq p$ and $s \leq b$. Assume that the control force generated by the input $\boldsymbol{u}$ has a symmetric property, namely, the real control forces $\boldsymbol{B}_{11} \boldsymbol{u}_{1}, \boldsymbol{B}_{12} \boldsymbol{u}_{2}, \boldsymbol{B}_{13} \boldsymbol{u}_{3}$, generated by the components $\boldsymbol{u}_{1}, \boldsymbol{u}_{2}, \boldsymbol{u}_{3}$ upon $\boldsymbol{x}_{1}$ are the same as those by $\boldsymbol{S}_{m} \boldsymbol{u}_{3}, \boldsymbol{u}_{2}, \boldsymbol{S}_{m} \boldsymbol{u}_{1}$, upon $\boldsymbol{S}_{p} \boldsymbol{x}_{3}$, and the control force by $\boldsymbol{u}_{1}$ upon $\boldsymbol{x}_{2}$ is the same as that by $\boldsymbol{S}_{m} \boldsymbol{u}_{3}$ upon $\boldsymbol{x}_{2}$. Therefore, the control matrix is in the 
form of

$$
\boldsymbol{B}=\left[\begin{array}{ccc}
\boldsymbol{B}_{11} & \boldsymbol{B}_{12} & \boldsymbol{B}_{13} \\
\boldsymbol{B}_{21} & \boldsymbol{B}_{22} & \boldsymbol{B}_{21} \boldsymbol{S}_{m} \\
\boldsymbol{S}_{p} \boldsymbol{B}_{13} \boldsymbol{S}_{m} & \boldsymbol{S}_{p} \boldsymbol{B}_{12} & \boldsymbol{S}_{p} \boldsymbol{B}_{11} \boldsymbol{S}_{m}
\end{array}\right],
$$

where $\boldsymbol{S}_{p}$ and $\boldsymbol{S}_{m}$ are $(p \times p)$ - and $(m \times m)$-matrices, respectively, as described in the expression (2).

In practice, it is difficult to implement the control high dimensional system since too many generalized coordinates need to be observed. Therefore, the high dimensional generalized coordinates of the structure, $\boldsymbol{x}$, are often required to be condensed to a low dimensional argument $\boldsymbol{y}$, which can be easily measured by properly installed sensors. There are $r$ sensors on the symmetric plane and $l$ sensors symmetrically located in each side of the symmetric plane, where $m \leq l \leq p$ and $s \leq r \leq b$. The coordinates measured by those sensors are denoted by $\boldsymbol{y}_{2}, \boldsymbol{y}_{1}$ and $\boldsymbol{y}_{3}$, respectively. Hence, the coordinates for the whole structure are expressed by $\boldsymbol{y}=\left[\boldsymbol{y}_{1}, \boldsymbol{y}_{2}, \boldsymbol{y}_{3}\right]^{\mathrm{T}}$. To maintain the symmetry of the condensed system, the condensation relation is given as

$$
\boldsymbol{x}=\left[\begin{array}{l}
\boldsymbol{x}_{1} \\
\boldsymbol{x}_{2} \\
\boldsymbol{x}_{3}
\end{array}\right]=\left[\begin{array}{ccc}
\boldsymbol{C}_{11} & \boldsymbol{C}_{21} & \boldsymbol{S}_{p} \boldsymbol{C}_{31} \boldsymbol{S}_{l} \\
\boldsymbol{C}_{21} & \boldsymbol{C}_{22} & \boldsymbol{C}_{21} \boldsymbol{S}_{l} \\
\boldsymbol{C}_{31} & \boldsymbol{S}_{p} \boldsymbol{C}_{21} & \boldsymbol{S}_{p} \boldsymbol{C}_{11} \boldsymbol{S}_{l}
\end{array}\right]\left[\begin{array}{l}
\boldsymbol{y}_{1} \\
\boldsymbol{y}_{2} \\
\boldsymbol{y}_{3}
\end{array}\right]=\boldsymbol{C y}
$$

Substituting Eq.(4) into Eq.(1) and pre-multiplying by $C^{\mathrm{T}}$ on the both sides of the equation yield a $(2 l+r)$-dimensional control system,

$$
\begin{aligned}
& \tilde{\boldsymbol{M}} \ddot{\boldsymbol{y}}+\tilde{\boldsymbol{K}} \boldsymbol{y}+\tilde{\boldsymbol{B}} \boldsymbol{u}=\mathbf{0}, \\
& \tilde{\boldsymbol{K}}=\boldsymbol{C}^{\mathrm{T}} \boldsymbol{K} \boldsymbol{C}=\left[\begin{array}{ccc}
\tilde{\boldsymbol{K}}_{11} & \tilde{\boldsymbol{K}}_{12} & \tilde{\boldsymbol{K}}_{13} \\
\tilde{\boldsymbol{K}}_{12}^{\mathrm{T}} & \tilde{\boldsymbol{K}}_{22} & \tilde{\boldsymbol{K}}_{12}^{\mathrm{T}} \boldsymbol{S}_{l} \\
\tilde{\boldsymbol{K}}_{13}^{\mathrm{T}} & \boldsymbol{S}_{l} \tilde{\boldsymbol{K}}_{12} & \tilde{\boldsymbol{K}}_{11}^{\mathrm{T}}
\end{array}\right], \\
& \tilde{\boldsymbol{B}}=\boldsymbol{C}^{\mathrm{T}} \boldsymbol{B}=\left[\begin{array}{ccc}
\tilde{\boldsymbol{B}}_{11} & \tilde{\boldsymbol{B}}_{12} & \tilde{\boldsymbol{B}}_{13} \\
\tilde{\boldsymbol{B}}_{21} & \tilde{\boldsymbol{B}}_{22} & \tilde{\boldsymbol{B}}_{21} \boldsymbol{S}_{m} \\
\tilde{S}_{l} \tilde{\boldsymbol{B}}_{13} \boldsymbol{S}_{m} & \boldsymbol{S}_{l} \tilde{\boldsymbol{B}}_{12} & \boldsymbol{S}_{l} \tilde{\boldsymbol{B}}_{11} \boldsymbol{S}_{m}
\end{array}\right],
\end{aligned}
$$

and $\tilde{\boldsymbol{K}}_{11}^{\mathrm{T}}=\tilde{\boldsymbol{K}}_{11}, \tilde{\boldsymbol{K}}_{13}^{\tilde{\mathrm{T}}}=\tilde{\boldsymbol{K}}_{13}, \tilde{\boldsymbol{K}}_{22}^{\mathrm{T}}=\tilde{\boldsymbol{K}}_{22} . \tilde{\boldsymbol{M}}$ has the same form as $\tilde{\boldsymbol{K}}$. Thus, $\tilde{\boldsymbol{M}}, \tilde{\boldsymbol{K}}$ and $\tilde{\boldsymbol{B}}$ of the condensed system retain the feature of the symmetric system, similar to those displayed by the expressions (2) and (3).

To conduct a reduction approach for Eq.(5), take coordinate transformations:

$$
\begin{aligned}
& \boldsymbol{y}=\left[\begin{array}{l}
\boldsymbol{y}_{1} \\
\boldsymbol{y}_{2} \\
\boldsymbol{y}_{3}
\end{array}\right]=\frac{1}{\sqrt{2}}\left[\begin{array}{ccc}
\boldsymbol{I}_{l} & \mathbf{0} & \boldsymbol{I}_{l} \\
\mathbf{0} & \sqrt{2} \boldsymbol{I}_{r} & \mathbf{0} \\
\boldsymbol{S}_{l} & \mathbf{0} & -\boldsymbol{S}_{l}
\end{array}\right]\left[\begin{array}{l}
\boldsymbol{q}_{1} \\
\boldsymbol{q}_{2} \\
\boldsymbol{q}_{3}
\end{array}\right]=\boldsymbol{H}_{l} \boldsymbol{q}, \\
& \boldsymbol{u}=\left[\begin{array}{l}
\boldsymbol{u}_{1} \\
\boldsymbol{u}_{2} \\
\boldsymbol{u}_{3}
\end{array}\right]=\frac{1}{\sqrt{2}}\left[\begin{array}{ccc}
\boldsymbol{I}_{m} & \mathbf{0} & \boldsymbol{I}_{m} \\
\mathbf{0} & \sqrt{2} \boldsymbol{I}_{s} & \mathbf{0} \\
\boldsymbol{S}_{m} & \mathbf{0} & -\boldsymbol{S}_{m}
\end{array}\right]\left[\begin{array}{l}
\boldsymbol{v}_{1} \\
\boldsymbol{v}_{2} \\
\boldsymbol{v}_{3}
\end{array}\right]=\boldsymbol{H}_{2} \boldsymbol{v},
\end{aligned}
$$

where $\boldsymbol{I}_{l}$ is an $l$-dimensional identity matrix. It should be noted that $\boldsymbol{H}_{i}^{\mathrm{T}} \boldsymbol{H}_{i}=\boldsymbol{I}, i=1,2$.

Substituting the expressions (7) and (8) into Eq.(5) and pre-multiplying it by $\boldsymbol{H}_{i}^{\mathrm{T}}$ lead to

$$
\begin{aligned}
& {\left[\begin{array}{cc}
\tilde{\boldsymbol{M}}_{11}+\tilde{\boldsymbol{M}}_{13} \boldsymbol{S}_{l} & \sqrt{2} \tilde{\boldsymbol{M}}_{12} \\
\sqrt{2} \tilde{\boldsymbol{M}}_{12}^{\mathrm{T}} & \tilde{\boldsymbol{M}}_{22}
\end{array}\right]\left[\begin{array}{c}
\ddot{\boldsymbol{q}}_{1} \\
\ddot{\boldsymbol{q}}_{2}
\end{array}\right]+\left[\begin{array}{cc}
\tilde{\boldsymbol{K}}_{11}+\tilde{\boldsymbol{K}}_{13} \boldsymbol{S}_{l} & \sqrt{2} \tilde{\boldsymbol{K}}_{12} \\
\sqrt{2} \tilde{\boldsymbol{K}}_{12}^{\mathrm{T}} & \tilde{\boldsymbol{K}}_{22}
\end{array}\right]\left[\begin{array}{l}
\boldsymbol{q}_{1} \\
\boldsymbol{q}_{2}
\end{array}\right]} \\
& +\left[\begin{array}{cc}
\tilde{\boldsymbol{B}}_{11}+\tilde{\boldsymbol{B}}_{13} \boldsymbol{S}_{m} & \sqrt{2} \tilde{\boldsymbol{B}}_{12} \\
\sqrt{2} \tilde{\boldsymbol{B}}_{13}^{\mathrm{T}} & \tilde{\boldsymbol{B}}_{22}
\end{array}\right]\left[\begin{array}{l}
\boldsymbol{v}_{1} \\
\boldsymbol{v}_{2}
\end{array}\right]=\mathbf{0} \\
& {\left[\tilde{\boldsymbol{M}}_{11}-\tilde{\boldsymbol{M}}_{13} \boldsymbol{S}_{l}\right] \ddot{\boldsymbol{q}}_{3}+\left[\tilde{\boldsymbol{K}}_{11}-\tilde{\boldsymbol{K}}_{13} \boldsymbol{S}_{l}\right] \boldsymbol{q}_{3}+\left[\tilde{\boldsymbol{B}}_{11}-\tilde{\boldsymbol{B}}_{13} \boldsymbol{S}_{m}\right] \boldsymbol{v}_{3}=\mathbf{0} \text {. }}
\end{aligned}
$$


So far, the original $(2 l+r)$-dimensional control system (5) has been reduced to one $(l+r)$ dimensional system and one $l$-dimensional system.

If the generalized coordinates are not set on the symmetric plane, the reduction may be even simpler. In this case $\boldsymbol{x}_{2}, \boldsymbol{u}_{2}, \boldsymbol{y}_{2}$ will be all $\mathbf{0}$ vectors, the expression (6) becomes

$$
\tilde{\boldsymbol{M}}=\left[\begin{array}{cc}
\tilde{\boldsymbol{M}}_{11} & \tilde{\boldsymbol{M}}_{13} \\
\tilde{\boldsymbol{M}}_{13}^{\mathrm{T}} & \tilde{\boldsymbol{M}}_{11}^{\tilde{\mathrm{T}}}
\end{array}\right], \quad \tilde{\boldsymbol{K}}=\left[\begin{array}{cc}
\tilde{\boldsymbol{K}}_{11} & \tilde{\boldsymbol{K}}_{13} \\
\tilde{\boldsymbol{K}}_{13}^{\mathrm{T}} & \tilde{\boldsymbol{K}}_{11}^{\tilde{\mathrm{T}}}
\end{array}\right], \quad \tilde{\boldsymbol{B}}=\left[\begin{array}{cc}
\tilde{\boldsymbol{B}}_{11} & \tilde{\boldsymbol{B}}_{13} \\
\boldsymbol{S}_{l} \tilde{\boldsymbol{B}}_{13} \boldsymbol{S}_{m} & \boldsymbol{S}_{l} \tilde{\boldsymbol{B}}_{11} \boldsymbol{S}_{m}
\end{array}\right],
$$

where $\tilde{\boldsymbol{M}}$ and $\tilde{\boldsymbol{K}}$ are symmetric with respect to both the main diagonal and the second diagonal entities of the matrices. Transformations of Eqs.(7) and (8) lead to

$$
\begin{aligned}
& \boldsymbol{y}=\left[\begin{array}{l}
\boldsymbol{y}_{1} \\
\boldsymbol{y}_{3}
\end{array}\right]=\frac{1}{\sqrt{2}}\left[\begin{array}{cc}
\boldsymbol{I}_{l} & \boldsymbol{I}_{l} \\
\boldsymbol{S}_{l} & -\boldsymbol{S}_{l}
\end{array}\right]\left[\begin{array}{l}
\boldsymbol{q}_{1} \\
\boldsymbol{q}_{3}
\end{array}\right]=\boldsymbol{U}_{l} \boldsymbol{q}, \\
& \boldsymbol{u}=\left[\begin{array}{l}
\boldsymbol{u}_{1} \\
\boldsymbol{u}_{3}
\end{array}\right]=\frac{1}{\sqrt{2}}\left[\begin{array}{cc}
\boldsymbol{I}_{m} & \boldsymbol{I}_{m} \\
\boldsymbol{S}_{m} & -\boldsymbol{S}_{m}
\end{array}\right]\left[\begin{array}{l}
\boldsymbol{v}_{1} \\
\boldsymbol{v}_{3}
\end{array}\right]=\boldsymbol{U}_{2} \boldsymbol{v} .
\end{aligned}
$$

Consequently, the control system is reduced to two $l$-dimensional control systems,

$$
\begin{aligned}
& \left(\tilde{\boldsymbol{M}}_{11}+\tilde{\boldsymbol{M}}_{13} \boldsymbol{S}_{l}\right) \ddot{\boldsymbol{q}}_{1}+\left(\tilde{\boldsymbol{K}}_{11}+\tilde{\boldsymbol{K}}_{13} \boldsymbol{S}_{l}\right) \boldsymbol{q}_{1}+\left(\tilde{\boldsymbol{B}}_{11}+\tilde{\boldsymbol{B}}_{13} \boldsymbol{S}_{m}\right) \boldsymbol{v}_{1}=\mathbf{0}, \\
& \left(\tilde{\boldsymbol{M}}_{11}-\tilde{\boldsymbol{M}}_{13} \boldsymbol{S}_{l}\right) \ddot{\boldsymbol{q}}_{3}+\left(\tilde{\boldsymbol{K}}_{11}-\tilde{\boldsymbol{K}}_{13} \boldsymbol{S}_{l}\right) \boldsymbol{q}_{3}+\left(\tilde{\boldsymbol{B}}_{11}-\tilde{\boldsymbol{B}}_{13} \boldsymbol{S}_{m}\right) \boldsymbol{v}_{3}=\mathbf{0} .
\end{aligned}
$$

From the expressions (12) and (13) as well as Eqs.(14) and (15), the vibration control of a real system can be implemented by the following procedure: (i) By virtue of the expression (12), transform the observed signal, $\boldsymbol{y}=\left\{\boldsymbol{y}_{1}, \boldsymbol{y}_{3}\right\}^{\mathrm{T}}$, of the whole system into $\boldsymbol{q}_{1}$ and $\boldsymbol{q}_{3}$ according to following formula:

$$
\boldsymbol{q}=\left[\begin{array}{l}
\boldsymbol{q}_{1} \\
\boldsymbol{q}_{3}
\end{array}\right]=\boldsymbol{U}_{1}^{\mathrm{T}} \boldsymbol{y}=\frac{1}{\sqrt{2}}\left[\begin{array}{l}
\boldsymbol{y}_{1}+\boldsymbol{S} \boldsymbol{y}_{3} \\
\boldsymbol{y}_{1}-\boldsymbol{S} \boldsymbol{y}_{3}
\end{array}\right] .
$$

(ii) Design feedback input $\boldsymbol{v}_{1}$ and $\boldsymbol{v}_{3}$ based on Eqs.(14) and (15) of the systems. (iii) Compose $\boldsymbol{v}_{1}$ and $\boldsymbol{v}_{3}$ to form the input of the whole system, $\boldsymbol{u}$, according to the expression (13). Thus, the vibration control of the original system with $2 l$-dimensions can be implemented by controlling two systems, each with $l$-dimensions.

\section{Reduction Approaches for Vibration Control of Cyclic Periodic Struc- tures}

A structure repeating with itself after a rotation of angle $\psi=2 \pi / n$ around a line is called an $n$-dimension cyclic periodic structure. Such a structure is composed of $n$ substructures whose shapes, physical properties, boundary conditions and mutual influences with other substructures are all identical. Let every substructure have $p$ generalized coordinates with the same label order. Let the generalized coordinates of the $i$ th substructure be $\boldsymbol{x}_{i}$, and $\boldsymbol{x}=\left[\boldsymbol{x}_{1}, \boldsymbol{x}_{2}, \cdots, \boldsymbol{x}_{n}\right]^{\mathrm{T}}$ be the generalized coordinates of the whole structure. The governing equation of the vibration of a cyclic periodic structure is given by

$$
M \ddot{x}+K \boldsymbol{x}+\boldsymbol{B u}=\mathbf{0},
$$

where $\boldsymbol{K}$ is a cyclic matrix,

$$
\boldsymbol{K}=\left[\begin{array}{ccccc}
\boldsymbol{K}_{11} & \boldsymbol{K}_{12} & \cdots & \boldsymbol{K}_{1 n-1} & \boldsymbol{K}_{1 n} \\
\boldsymbol{K}_{1 n} & \boldsymbol{K}_{12} & \cdots & \boldsymbol{K}_{1 n-2} & \boldsymbol{K}_{1 n-1} \\
\vdots & \vdots & \vdots & \vdots & \vdots \\
\boldsymbol{K}_{12} & \boldsymbol{K}_{13} & \cdots & \boldsymbol{K}_{1 n} & \boldsymbol{K}_{11}
\end{array}\right]
$$


with $\boldsymbol{K}_{11}^{\mathrm{T}}=\boldsymbol{K}_{11}, \boldsymbol{K}_{1 a}^{\mathrm{T}}=\boldsymbol{K}_{1(n+2-a)}, a=2,3, \cdots, n$, and $\boldsymbol{M}$ having the same form as $\boldsymbol{K}$.

Make the numbers and locations of actuators in every substructure identical. The component $\boldsymbol{u}_{j}$ of the system input $\boldsymbol{u}=\left[\boldsymbol{u}_{1}, \boldsymbol{u}_{2}, \cdots, \boldsymbol{u}_{n}\right]^{\mathrm{T}}$ is an $n$-dimensional vector. The relationship between $\boldsymbol{u}_{j}$ and the control force of the substructures possesses cyclic periodic properties; that is, the control forces $\boldsymbol{B}_{11} \boldsymbol{u}_{1}, \boldsymbol{B}_{12} \boldsymbol{u}_{2}, \cdots, \boldsymbol{B}_{1 n-1} \boldsymbol{u}_{n-1}, \boldsymbol{B}_{1 n} \boldsymbol{u}_{n}$ generated by $\boldsymbol{u}_{1}, \boldsymbol{u}_{2}, \cdots, \boldsymbol{u}_{n-1}, \boldsymbol{u}_{n}$ upon $\boldsymbol{x}_{1}$ are the same as those generated by $\boldsymbol{u}_{2}, \cdots, \boldsymbol{u}_{n-1}, \boldsymbol{u}_{n}, \boldsymbol{u}_{1}$ upon $\boldsymbol{x}_{2}$. This analogy can also be applied to the rest terms. Therefore, the control matrix has the following form:

$$
\boldsymbol{B}=\left[\begin{array}{ccccc}
\boldsymbol{B}_{11} & \boldsymbol{B}_{12} & \cdots & \boldsymbol{B}_{1 n-1} & \boldsymbol{B}_{1 n} \\
\boldsymbol{B}_{1 n} & \boldsymbol{B}_{11} & \cdots & \boldsymbol{B}_{1 n-2} & \boldsymbol{B}_{1 n-1} \\
\vdots & \vdots & \vdots & \vdots & \vdots \\
\boldsymbol{B}_{12} & \boldsymbol{B}_{13} & \cdots & \boldsymbol{B}_{1 n} & \boldsymbol{B}_{11}
\end{array}\right] .
$$

For cyclic structures with a large number degree of freedom, condensation of its generalized coordinates is often needed. High dimensional generalized coordinates $\boldsymbol{x}$ are condensed into a low dimensional argument $\boldsymbol{y}$, which can be observed by sensors more easily. To keep the condensed system remain as cyclic periodic, equal number $(l)$ of sensors with the same type and location are mounted in every substructures. Denote the observed value of the $i$ th substructure by $\boldsymbol{y}_{i}$, and that of the whole structure by $\boldsymbol{y}=\left[\boldsymbol{y}_{1}, \boldsymbol{y}_{2}, \cdots, \boldsymbol{y}_{n}\right]^{\mathrm{T}}$. The condensation relation is given as

$$
\boldsymbol{x}=\left[\begin{array}{c}
\boldsymbol{x}_{1} \\
\boldsymbol{x}_{2} \\
\vdots \\
\boldsymbol{x}_{n}
\end{array}\right]=\left[\begin{array}{cccc}
\boldsymbol{C}_{11} & \boldsymbol{C}_{n 1} & \cdots & \boldsymbol{C}_{21} \\
\boldsymbol{C}_{21} & \boldsymbol{C}_{11} & \cdots & \boldsymbol{C}_{31} \\
\vdots & \vdots & \vdots & \vdots \\
\boldsymbol{C}_{n 1} & \boldsymbol{C}_{n-1,1} & \cdots & \boldsymbol{C}_{11}
\end{array}\right]\left[\begin{array}{c}
\boldsymbol{y}_{1} \\
\boldsymbol{y}_{2} \\
\vdots \\
\boldsymbol{y}_{n}
\end{array}\right]=\boldsymbol{C} \boldsymbol{y}
$$

Substituting this expression into Eq.(17) and pre-multiplying this equation by $\boldsymbol{C}^{\mathrm{T}}$ yield the equation of vibration control of $(n l)$-dimensional system,

$$
\tilde{\boldsymbol{M}} \ddot{\boldsymbol{y}}+\tilde{\boldsymbol{K}} \boldsymbol{y}+\tilde{\boldsymbol{B}} \boldsymbol{u}=\mathbf{0},
$$

where $\tilde{\boldsymbol{K}}, \tilde{\boldsymbol{M}}$, and $\tilde{\boldsymbol{B}}$ are all cyclic matrices, while $\tilde{\boldsymbol{K}}$ and $\tilde{\boldsymbol{M}}$ are also symmetric matrices.

By virtue of the properties of these matrices, and taking proper transformation, we can reduce the dimension of the system (21). The transformation is given as

$$
\begin{aligned}
& \boldsymbol{y}=\left[\begin{array}{c}
\boldsymbol{y}_{1} \\
\boldsymbol{y}_{2} \\
\vdots \\
\boldsymbol{y}_{n}
\end{array}\right]=\frac{1}{\sqrt{n}}\left[\begin{array}{cccc}
\boldsymbol{I}_{l} & \boldsymbol{I}_{l} & \cdots & \boldsymbol{I}_{l} \\
\mathrm{e}^{\mathrm{i} \psi} \boldsymbol{I}_{l} & \mathrm{e}^{\mathrm{i} 2 \psi} \boldsymbol{I}_{l} & \cdots & \mathrm{e}^{\mathrm{i} n \psi} \boldsymbol{I}_{l} \\
\vdots & \vdots & \vdots & \vdots \\
\mathrm{e}^{\mathrm{i}(n-1) \psi} \boldsymbol{I}_{l} & \mathrm{e}^{\mathrm{i} 2(n-1) \psi} \boldsymbol{I}_{l} & \cdots & \mathrm{e}^{\mathrm{i} n(n-1) \psi} \boldsymbol{I}_{l}
\end{array}\right]\left[\begin{array}{c}
\boldsymbol{q}_{1} \\
\boldsymbol{q}_{2} \\
\vdots \\
\boldsymbol{q}_{n}
\end{array}\right]=\boldsymbol{U}_{1} \boldsymbol{q}, \\
& \boldsymbol{u}=\left[\begin{array}{llll}
\boldsymbol{u}_{1}, & \boldsymbol{u}_{2}, & \cdots, & \boldsymbol{u}_{n}
\end{array}\right]^{\mathrm{T}}=\boldsymbol{U}_{2}\left[\begin{array}{llll}
\boldsymbol{v}_{1}, & \boldsymbol{v}_{2}, & \cdots, & \boldsymbol{v}_{n}
\end{array}\right]^{\mathrm{T}}=\boldsymbol{U}_{2} \boldsymbol{v},
\end{aligned}
$$

where $\mathrm{i}=\sqrt{-1}, \psi=2 \pi / n$; matrix $\boldsymbol{U}_{2}$ has the similar form as $\boldsymbol{U}_{1}$ with the identity matrix $\boldsymbol{I}_{l}$ being replaced by $\boldsymbol{I}_{m}$. It should be noted that $\overline{\boldsymbol{U}}_{j}^{\mathrm{T}} \boldsymbol{U}_{j}=\boldsymbol{I}, j=1,2$.

Substituting the transformations (22) and (23) into Eq.(21) and pre-multiplying it by $\overline{\boldsymbol{U}}_{1}^{\mathrm{T}}$ yield

$$
\begin{aligned}
& \overline{\boldsymbol{U}}_{1}^{\mathrm{T}} \tilde{\boldsymbol{K}} \boldsymbol{U}_{1}=\operatorname{diag}\left[\boldsymbol{K}_{1}, \quad \boldsymbol{K}_{2}, \quad \cdots, \quad \boldsymbol{K}_{n}\right], \quad \boldsymbol{K}_{s}=\sum_{j=1}^{n} \tilde{\boldsymbol{K}}_{1 j} \mathrm{e}^{\mathrm{i}(j-1) s \psi}, \\
& \overline{\boldsymbol{U}}_{1}^{\mathrm{T}} \tilde{\boldsymbol{B}} \boldsymbol{U}_{2}=\operatorname{diag}\left[\begin{array}{llll}
\boldsymbol{B}_{1}, & \boldsymbol{B}_{2}, & \cdots, & \boldsymbol{B}_{n}
\end{array}\right], \quad \boldsymbol{B}_{s}=\sum_{j=1}^{n} \tilde{\boldsymbol{B}}_{1 j} \mathrm{e}^{\mathrm{i}(j-1) s \psi},
\end{aligned}
$$


where $\overline{\boldsymbol{K}}_{s}^{\mathrm{T}}=\boldsymbol{K}_{s}, \overline{\boldsymbol{K}}_{n-s}=\boldsymbol{K}_{s}, \overline{\boldsymbol{B}}_{n-s}=\boldsymbol{B}_{s}$.

Finally, Eq.(21) are reduced to $n$ equations of vibration control of an $l$-dimensional system:

$$
\boldsymbol{M}_{s} \ddot{\boldsymbol{q}}_{s}+\boldsymbol{K}_{s} \boldsymbol{q}_{s}+\boldsymbol{B}_{s} \boldsymbol{v}_{s}=\mathbf{0}, \quad s=1,2, \cdots, n .
$$

Denoting the real and imaginary parts of matrix in Eq.(26), the equation is recast into the following form:

$$
\left[\begin{array}{cc}
\boldsymbol{M}_{s}^{\mathrm{r}} & -\boldsymbol{M}_{s}^{\mathrm{i}} \\
\boldsymbol{M}_{s}^{\mathrm{i}} & \boldsymbol{M}_{s}^{\mathrm{r}}
\end{array}\right]\left[\begin{array}{c}
\ddot{\boldsymbol{q}}_{s}^{\mathrm{r}} \\
\ddot{\boldsymbol{q}}_{s}^{\mathrm{i}}
\end{array}\right]+\left[\begin{array}{cc}
\boldsymbol{K}_{s}^{\mathrm{r}} & -\boldsymbol{K}_{s}^{\mathrm{i}} \\
\boldsymbol{K}_{s}^{\mathrm{i}} & \boldsymbol{K}_{s}^{\mathrm{r}}
\end{array}\right]\left[\begin{array}{c}
\boldsymbol{q}_{s}^{\mathrm{r}} \\
\boldsymbol{q}_{s}^{\mathrm{i}}
\end{array}\right]+\left[\begin{array}{cc}
\boldsymbol{B}_{s}^{\mathrm{r}} & -\boldsymbol{B}_{s}^{\mathrm{i}} \\
\boldsymbol{B}_{s}^{\mathrm{i}} & \boldsymbol{B}_{s}^{\mathrm{r}}
\end{array}\right]\left[\begin{array}{c}
\boldsymbol{v}_{s}^{\mathrm{r}} \\
\boldsymbol{v}_{s}^{\mathrm{i}}
\end{array}\right]=\mathbf{0}, \quad s=1,2, \cdots, n .
$$

For $s=n$ and $n / 2$ (if $n$ is even), $\boldsymbol{K}_{s}, \boldsymbol{M}_{s}$ and $\boldsymbol{B}_{s}$ appeared in Eq.(27) are real matrices, and their imaginary parts are zero. For other values of $s, \boldsymbol{K}_{s}, \boldsymbol{M}_{s}$ and $\boldsymbol{B}_{s}$ are all complex matrices. However, since these three matrices with subscripts $s$ and $(n-s)$ are conjugate respectively, as seen in the expressions (24) and (25), the solutions to the $s$ th and $(n-s)$ th equations in Eq.(26) are conjugate, too; thus we have $\boldsymbol{q}_{n-s}=\overline{\boldsymbol{q}}_{s}, \boldsymbol{v}_{n-s}=\overline{\boldsymbol{v}}_{s}$.

Therefore, the original control system (21) with $(n l)$-degrees of freedom has been reduced to a control system (27) composed of one subsystem (or two subsystems) with $l$-degrees of freedom and $(n-1) / 2($ or $(n-2) / 2)$ subsystems with $(2 l)$-degrees of freedom. Substituting $\boldsymbol{q}_{s}^{\mathrm{r}}, \boldsymbol{q}_{s}^{\mathrm{i}}, \boldsymbol{v}_{s}^{\mathrm{r}}$, $\boldsymbol{v}_{s}^{\mathrm{i}}$ solved from Eq.(27) into the transformations (22) and (23) yields the solution $\boldsymbol{y}=\boldsymbol{y}^{\mathrm{r}}+\mathrm{i} \boldsymbol{y}^{\mathrm{i}}$ and $\boldsymbol{u}=\boldsymbol{u}^{\mathrm{r}}+\mathrm{i} \boldsymbol{u}^{\mathrm{i}}$ for the original system. It can be verified that $\boldsymbol{y}^{\mathrm{i}}=\mathbf{0}$ and $\boldsymbol{u}^{\mathrm{i}}=\mathbf{0}$. Hence, the solution of the original system is real.

It is therefore suggested that the vibration control of the system (21) can be implemented by the following procedure: (i) By virtue of the expression (21), the observed signal $\boldsymbol{y}$ of the original system is separated into several subsystems according to the following expressions:

$$
\begin{aligned}
\boldsymbol{q} & =\overline{\boldsymbol{U}}_{1}^{\mathrm{T}} \boldsymbol{y}, \\
\boldsymbol{q}_{s}^{\mathrm{r}} & =\frac{1}{\sqrt{n}}\left[\begin{array}{llll}
\boldsymbol{I}, & \cos s \psi \boldsymbol{I}, & \cdots, & \cos (n-1) s \psi \boldsymbol{I}
\end{array}\right]\left[\begin{array}{llll}
\boldsymbol{y}_{1}, & \boldsymbol{y}_{2}, & \cdots, & \boldsymbol{y}_{n}
\end{array}\right]^{\mathrm{T}}, \\
\boldsymbol{q}_{s}^{\mathrm{i}} & =\frac{1}{\sqrt{n}}\left[\begin{array}{lllll}
\mathbf{0}, & -\sin s \psi \boldsymbol{I}, & \cdots, & -\sin (n-1) s \psi \boldsymbol{I}
\end{array}\right]\left[\begin{array}{llll}
\boldsymbol{y}_{1}, & \boldsymbol{y}_{2}, & \cdots, & \boldsymbol{y}_{n}
\end{array}\right]^{\mathrm{T}} .
\end{aligned}
$$

(ii) Inputs $\boldsymbol{v}_{s}^{\mathrm{r}}, \boldsymbol{v}_{s}^{\mathrm{i}}$ are designed according to the subsystems (27). (iii) Inputs $\boldsymbol{u}$ of the system (21) are composed of $\boldsymbol{v}_{s}$ according to Eq.(23). Consequently, the (nl)-dimensional control system (21) is reduced to control systems (27), which is one (or two) $l$-dimensional subsystem and $(n-1) / 2($ or $(n-2) / 2$ for even $n)(2 l)$-dimensional subsystems.

\section{Reduction Approaches for Vibration Control of Linking Structures}

A linking structure refers to an assembly of identical substructures forming a chain linking between two fixed ends. Each substructure of the linking structure is influenced only by its two neighboring substructures, and these two influences are symmetric. If $p$ generalized coordinates at the same location in each substructure are taken as degrees of freedom, then the stiffness matrix of the whole structure composed by $n$ substructures will have the block form:

$$
\boldsymbol{K}=\left[\begin{array}{ccccc}
\boldsymbol{K}_{11} & \boldsymbol{K}_{12} & & & \\
\boldsymbol{K}_{12} & \boldsymbol{K}_{11} & \boldsymbol{K}_{12} & & \\
& \ddots & \ddots & \ddots & \\
& & \boldsymbol{K}_{12} & \boldsymbol{K}_{11} & \boldsymbol{K}_{12} \\
& & & \boldsymbol{K}_{12} & \boldsymbol{K}_{11}
\end{array}\right]
$$

where $\boldsymbol{K}_{11}^{\mathrm{T}}=\boldsymbol{K}_{11}, \boldsymbol{K}_{12}^{\mathrm{T}}=\boldsymbol{K}_{12}$. Mass matrix $\boldsymbol{M}$ has the similar form as $\boldsymbol{K}$. 
When the actuators are placed at the same location in every substructure, the input of the system is $\boldsymbol{u}=\left[\boldsymbol{u}_{1}, \cdots, \boldsymbol{u}_{n}\right]^{\mathrm{T}}$. The $m$-dimensional component vector $\boldsymbol{u}_{j}$ generates a control force $\boldsymbol{B}_{11} \boldsymbol{u}_{j}$ for the $j$ th substructure and a symmetric control force $\boldsymbol{B}_{12} \boldsymbol{u}_{j}$ for the $(j-1)$ th and the $(j+1)$ th substructures, respectively. However, $\boldsymbol{u}_{j}$ does not generate any control force acting on other substructures. Now the whole structure has $(n p)$-degrees of freedom, and the equation of vibration control is given by

$$
\boldsymbol{M} \ddot{x}+\boldsymbol{K} \boldsymbol{x}+\boldsymbol{B} \boldsymbol{u}=\mathbf{0},
$$

where $\boldsymbol{B}$ has the similar form with $\boldsymbol{K}$ in Eq.(28).

Again, we condense the high dimensional generalized coordinates $\boldsymbol{x}$ into low dimensional argument $\boldsymbol{y}$ in order to make the control more practical. To keep the condensed system remain as a linking system, $l$ identical sensors are placed in the same location of all the substructures. Denote the observed value of the $i$ th substructure by $\boldsymbol{y}_{i}$, and that of the whole system is denoted by $\boldsymbol{y}=\left[\boldsymbol{y}_{1}, \boldsymbol{y}_{2}, \cdots, \boldsymbol{y}_{n}\right]^{\mathrm{T}}$. It is assumed that $\boldsymbol{x}_{i}$ is condensed to $\boldsymbol{y}_{i}$ only without any relations with other $\boldsymbol{y}$ s and the same condensing rule is applied through $i=1, \cdots, n$. Thus,

$$
\boldsymbol{x}=\left[\begin{array}{c}
\boldsymbol{x}_{1} \\
\boldsymbol{x}_{2} \\
\vdots \\
\boldsymbol{x}_{n}
\end{array}\right]=\left[\begin{array}{llll}
\boldsymbol{C}_{11} & & & \\
& \boldsymbol{C}_{11} & & \\
& & \ddots & \\
& & & \boldsymbol{C}_{11}
\end{array}\right]\left[\begin{array}{c}
\boldsymbol{y}_{1} \\
\boldsymbol{y}_{2} \\
\vdots \\
\boldsymbol{y}_{n}
\end{array}\right]=\boldsymbol{C y}
$$

Substituting Eq.(30) into Eq.(29) and pre-multiplying it by $\boldsymbol{C}^{\mathrm{T}}$ yield the equation of vibration control of the $(n l)$-dimensional system,

$$
\tilde{\boldsymbol{M}} \ddot{\boldsymbol{y}}+\tilde{\boldsymbol{K}} \boldsymbol{y}+\tilde{\boldsymbol{B}} \boldsymbol{u}=\mathbf{0}
$$

where $\tilde{\boldsymbol{M}}, \tilde{\boldsymbol{K}}$ and $\tilde{\boldsymbol{B}}$ have similar forms with $\boldsymbol{K}$.

$$
\begin{aligned}
& \tilde{\boldsymbol{M}}_{11}=\boldsymbol{C}_{11}^{\mathrm{T}} \boldsymbol{M}_{11} \boldsymbol{C}_{11}=\tilde{\boldsymbol{M}}_{11}^{\mathrm{T}}, \quad \tilde{\boldsymbol{M}}_{12}=\boldsymbol{C}_{11}^{\mathrm{T}} \boldsymbol{M}_{12} \boldsymbol{C}_{11}=\tilde{\boldsymbol{M}}_{12}^{\mathrm{T}}, \quad \tilde{\boldsymbol{K}}_{11}=\boldsymbol{C}_{11}^{\mathrm{T}} \boldsymbol{K}_{11} \boldsymbol{C}_{11}=\tilde{\boldsymbol{K}}_{11}^{\mathrm{T}}, \\
& \tilde{\boldsymbol{K}}_{12}=\boldsymbol{C}_{11}^{\mathrm{T}} \tilde{\boldsymbol{K}}_{12} \boldsymbol{C}_{11}=\tilde{\boldsymbol{K}}_{12}^{\mathrm{T}}, \quad \tilde{\boldsymbol{B}}_{11}=\boldsymbol{C}_{11}^{\mathrm{T}} \boldsymbol{B}_{11}, \quad \tilde{\boldsymbol{B}}_{12}=\boldsymbol{C}_{11}^{\mathrm{T}} \boldsymbol{B}_{12} .
\end{aligned}
$$

Using the properties of matrices $\tilde{\boldsymbol{M}}, \tilde{\boldsymbol{K}}$ and $\tilde{\boldsymbol{B}}$, and applying proper coordinate transformations, we can reduce Eq.(31) into $n$ control systems with $l$-degrees of freedom. Let $\phi=\pi /(n+1)$, and apply the transformation

$$
\begin{aligned}
& \boldsymbol{y}=\left[\begin{array}{c}
\boldsymbol{y}_{1} \\
\boldsymbol{y}_{2} \\
\vdots \\
\boldsymbol{y}_{n}
\end{array}\right]=\sqrt{\frac{2}{n+1}}\left[\begin{array}{cccc}
\sin \phi \boldsymbol{I}_{l} & \sin 2 \phi \boldsymbol{I}_{l} & \cdots & \sin n \phi \boldsymbol{I}_{l} \\
\sin 2 \phi \boldsymbol{I}_{l} & \sin 4 \phi \boldsymbol{I}_{l} & \cdots & \sin 2 n \phi \boldsymbol{I}_{l} \\
\vdots & \vdots & \vdots & \vdots \\
\sin n \phi \boldsymbol{I}_{l} & \sin 2 n \phi \boldsymbol{I}_{l} & \cdots & \sin n n \phi \boldsymbol{I}_{l}
\end{array}\right]\left[\begin{array}{c}
\boldsymbol{q}_{1} \\
\boldsymbol{q}_{2} \\
\vdots \\
\boldsymbol{q}_{n}
\end{array}\right]=\boldsymbol{R}_{1} \boldsymbol{q}, \\
& \boldsymbol{u}=\left[\begin{array}{llll}
\boldsymbol{u}_{1}, & \boldsymbol{u}_{2}, & \cdots, & \boldsymbol{u}_{n}
\end{array}\right]^{\mathrm{T}}=\boldsymbol{R}_{2} \boldsymbol{U} .
\end{aligned}
$$

Here $\boldsymbol{R}_{2}$ has the same form as $\boldsymbol{R}_{1}$ except for $\boldsymbol{I}_{l}$ being replaced by $\boldsymbol{I}_{m}$. Substituting Eqs.(32), (33) into Eq.(31) and pre-multiplying it by $\boldsymbol{R}_{1}^{\mathrm{T}}$ yield

$$
\begin{aligned}
& \boldsymbol{R}_{1}^{\mathrm{T}} \tilde{\boldsymbol{K}} \boldsymbol{R}_{1}=\operatorname{diag}\left[\begin{array}{llll}
\boldsymbol{K}_{1}, & \boldsymbol{K}_{2}, & \cdots, & \boldsymbol{K}_{n}
\end{array}\right], \quad \boldsymbol{K}_{s}=\tilde{\boldsymbol{K}}_{11}+2 \cos s \phi \tilde{\boldsymbol{K}}_{12}, \quad s=1,2, \cdots, n, \\
& \boldsymbol{R}_{1}^{\mathrm{T}} \tilde{\boldsymbol{M}} \boldsymbol{R}_{1}=\operatorname{diag}\left[\begin{array}{llll}
\boldsymbol{M}_{1}, & \boldsymbol{M}_{2}, & \cdots, & \boldsymbol{M}_{n}
\end{array}\right], \quad \boldsymbol{M}_{s}=\tilde{\boldsymbol{M}}_{11}+2 \cos s \phi \tilde{\boldsymbol{M}}_{12}, \\
& \boldsymbol{R}_{1}^{\mathrm{T}} \tilde{\boldsymbol{B}} \boldsymbol{R}_{2}=\operatorname{diag}\left[\begin{array}{llll}
\boldsymbol{B}_{1}, & \boldsymbol{B}_{2}, & \cdots, & \boldsymbol{B}_{n}
\end{array}\right], \quad \boldsymbol{B}_{s}=\tilde{\boldsymbol{B}}_{11}+2 \cos s \phi \tilde{\boldsymbol{B}}_{12} .
\end{aligned}
$$

Therefore, the control problem of the system (31) with $(n l)$-degrees of freedom is reduced to that of $n$ systems with $l$-degrees of freedom, i.e.,

$$
\boldsymbol{M}_{s} \ddot{\boldsymbol{q}}_{s}+\boldsymbol{K}_{s} \boldsymbol{q}_{s}+\boldsymbol{B}_{s} \boldsymbol{v}_{s}=\mathbf{0}, \quad s=1,2, \cdots, n .
$$


The vibration control of the system (31) can be implemented by following procedure: (i) Using the expression (32), resolve the observed $\boldsymbol{y}$ of the whole system into $\boldsymbol{q}_{s}$ according to the following expression $\boldsymbol{q}=\left[\boldsymbol{q}_{1}, \cdots, \boldsymbol{q}_{n}\right]^{\mathrm{T}}=\boldsymbol{R}_{1}^{\mathrm{T}} \boldsymbol{y}$. (ii) Input $\boldsymbol{q}_{s}$ into the subsystems (34), design feedback input $\boldsymbol{v}_{s}$ according to these subsystems. (iii) Compose $\boldsymbol{u}$ of $\boldsymbol{v}_{s}$ according to Eq.(33), and take it as the input of the whole system.

\section{Conclusions}

Reduction approaches for vibration control of symmetric, cyclic periodic and linking structures are presented in this paper. Using the presented reduction method, the vibration control problem of an entire repetitive system can be reduced to several vibration control problems of its substructures whose dimensions are much lower than that of the original system provided that the distribution of its actuators and sensors have the same repetitive properties as the structure itself. Moreover, the procedures to implement the vibration control of symmetric, cyclic periodic and linking structures are also elaborated, respectively.

As a special example, consider a case in which every substructure has one sensor and one actuator. By means of the reduction approaches presented in this paper, for symmetric structures, a double-input double-output control system can be reduced into two single-input single-output control systems. For $n$-dimensional cyclic period structures, an $n$-input $n$-output control system can be reduced into one (or two, if $n$ is even) single-input single-output control system and $(n-1) / 2$ (or $(n-2) / 2$, if $n$ is even) 2-input 2-output control systems. For linking structures, an $n$-input $n$-output control system can be reduced into $n$ single-input single-output control systems.

The procedures to implement the vibration control of real systems are also elaborated in this paper.

Some problems, such as the controllability, observability and system robustness problem caused by using the reduction approach, will be investigated in the future studies.

Acknowledgments The third author acknowledges the support from Beijing Institute of Spacecraft System Engineering, Chinese Academy of Space Technology.

\section{References}

[1] Evensen D A. Vibration analysis of multi-symmetric structures[J]. AIAA Journal, 1976, 14(4):446-453.

[2] Thomas D L. Dynamics of rotational periodic structures[J]. Internat J of Numerical Methods in Engineering, 1979, 14:81-102.

[3] Cai C, Cheung Y, Chan H. Uncoupling of dynamic equations for periodic structures[J]. J of Sound and Vibration, 1990, 139(2):253-263.

[4] Chan H, Cai C, Cheung Y. Exact Analysis of Structures with Periodicity Using UTransformation[M]. World Scientific Publication, Hong Kong, 1998.

[5] Wang Dajun, Wang C C. Natural vibration of repetitive structures[J]. Chinese J of Mechanics, 2000, 16(2):85-95.

[6] Wang Dajun, Zhou Chunyan, Jie Rong. Free and forced vibration of repetitive structures[J]. Internat J of Solids and Structures, 2003, 40:5477-5494.

[7] Bryson A E, Wiesinger F A. Modeling and Control of Flexible Vehicles in Space[R]. AD-A219622, 1990. 